\title{
ROBUST MODEL PREDICTIVE CONTROL FOR SWITCHED PIECEWISE LINEAR HYBRID SYSTEMS
}

\author{
Jean THOMAS \\ Faculty of Industrial Education, Beni-Sueif University, Egypt \\ Phone : +20(0)125263476_Email :jhh_thomas@yahoo.com
}

(Received September 17, 2006 Accepted October 15, 2006)

\begin{abstract}
This paper investigates the robust tracking and regulation control problems for discrete-time, switched piecewise linear hybrid systems affected by parameter variations. In particular, the main question addressed is related to the existence of a controller such that the closedloop system exhibits an attainable desired behavior under all possible parameter variation. Checking attainability and calculating the state space regions for which a robust control is assured despite the uncertainty is performed using a polyhedral approach. A model predictive control law derived from a quadratic cost function minimization is further examined as a simple and fast sub-optimal robust control. An application of the proposed technique to a two-tank benchmark is finally presented.
\end{abstract}

KEYWORDS: Piecewise Linear Systems, Robust Model Predictive Control, Model Uncertainty, Attainability.

\section{INTRODUCTION}

Hybrid systems are now of common use in many control applications in industry, e.g. in control of mechanical systems, process control, automotive industry, power systems, aircraft and traffic control. Hybrid systems are heterogeneous dynamical systems, their behavior is determined by interacting continuous variable and discrete event dynamics. Various approaches have been proposed to model hybrid systems [1], such as Automata, Petri nets, Linear Complementary (LC), Piecewise Affine (PWA) [2], Mixed Logical Dynamical (MLD) models [3]. Different techniques are used to control hybrid systems, for example Model Predictive Control (MPC) [4], [5], [6], [7] and optimal control [3].

An attractive and challenging field of research is currently dealing with hybrid systems subject to uncertainties, either parameters uncertainties or disturbances influences, where problems like safety, reachability, attainability and robust control become interesting questions for researchers. In this direction, this paper examines a class of uncertain discrete-time switched piecewise linear hybrid systems affected by parameter variations. For this class of systems, some solutions to the above mentioned problems are already proposed in the literature. For example, in [8], an attainability checking that employs the predecessor operator, and a controller technique using finite automata and 
linear programming is presented. In [9] and [10], a control technique based on minimizing the worst-case cost function (min-max problem) is proposed to solve the control problem.

The contribution of this paper is based on a simple polyhedral approach enabling the elaboration of the state space regions for which a robust control exists which drives the plant to a desired behavior despite the parameter variation. The safety, reachability and attainability questions are examined through this framework and a robust Model Predictive Control (MPC) with quadratic cost function is presented as a fast suboptimal robust control for the considered systems.

The paper is organized as follows. A brief description of switched piecewise linear hybrid systems and the related class is given in Section 2. Section 3 develops the polyhedral approach which will elaborate the state space regions where reachability, safety and attainability questions can be assured. A fast and suboptimal robust control is then developed in Section 4 for the considered class. An application of the proposed technique to a two-tank benchmark is presented in Section 5. Finally the conclusions and some remarks are given in Section 6.

\section{UNCERTAIN SWITCHED PIECEWISE LINEAR HYBRID SYSTEMS}

Switched piecewise linear/affine systems are powerful tools for describing or approximating both nonlinear and hybrid systems, and represent the easiest extension from linear to hybrid systems. This paper focuses on the particular class of uncertain discrete-time switched piecewise linear hybrid systems subject to parameter variations, defined as:

$$
\begin{aligned}
\Sigma^{i}:\left\{\mathbf{x}_{k+1}\right. & \left.=\mathbf{A}^{i}\left(w_{k}^{i}\right) \mathbf{x}_{k}+\mathbf{B}^{i}\left(w_{k}^{i}\right) \mathbf{u}_{k}\right\} \\
& \text { for }\left[\begin{array}{l}
\mathbf{x}_{k} \\
\mathbf{u}_{k}
\end{array}\right] \in \chi_{i}
\end{aligned}
$$

$\left\{\chi_{i}\right\}_{i=1}^{s}$ are the polyhedral validity domains in the state and input spaces, $s$ being the total number of possible modes. Each $\chi_{i}$ is given by:

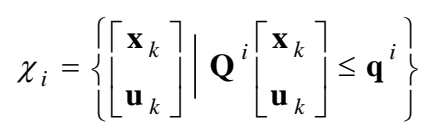

where $\mathbf{x}_{k} \in \mathbf{X}, \mathbf{u}_{k} \in \mathbf{U}, w_{k}^{i} \in \mathbf{W}^{i}$ are the system state, the input control and the uncertainty respectively at instant $k$ (for the $i^{\text {th }}$ model). It is assumed that $\mathbf{X}, \mathbf{U}$ and $\mathbf{W}^{i}(i \in I=(1,2, \cdots, s)$, where $I$ is the collection of all modes) are assigned polytopes.

Exact state measurement $\mathbf{x}$ is supposed to be available. Note that the sets $\chi_{i}$ are assumed here to be not disjoint so that the desired model dynamics can be chosen by the bias of switching (logical) decision variables.

Each sub-model $\Sigma^{i}$ is defined by the 4-uple $\left(\mathbf{A}^{i}, \mathbf{B}^{i}, \mathbf{Q}^{i}, \mathbf{q}^{i}\right)$, where 
$\mathbf{A}^{i} \in \mathfrak{R}^{n \times n}, \mathbf{B}^{i} \in \mathfrak{R}^{n \times m}, \mathbf{Q}^{i} \in \mathfrak{R}^{p_{i} \times(n+m)}$ and $\mathbf{q}^{i}$ is a suitable constant vector, where $n, m$ are respectively the number of states and inputs, and $p_{i}$ is the number of hyperplanes defining the $\chi_{i}$ polyhedral.

Taking into account uncertainty as it appears in Eq. 1, the following considers polytopic uncertainty in $\mathbf{A}^{i}\left(w^{i}\right)$ and $\mathbf{B}^{i}\left(w^{i}\right)$ for every mode $i \in I$. As a polyhedral set $\mathbf{R}$ can be represented either by a set of linear inequalities $\mathbf{R}=\{\mathbf{x} \mid \mathbf{F x} \leq \mathbf{g}\}$, or by its dual representation in terms of its vertex set $\left\{\mathbf{x}^{j}\right\}$, this polytopic uncertainty will be structured as follows:

$$
\mathbf{A}^{i}(w)=\sum_{j}^{v_{i}} w^{i j} \mathbf{A}^{i j}, \quad \mathbf{B}^{i}(w)=\sum_{j}^{v_{i}} w^{i j} \mathbf{B}^{i j}
$$

where $w^{i j} \geq 0$ and $\sum_{j=1}^{v} w^{i j}=1 .\left(\mathbf{A}^{i j}, \mathbf{B}^{i j}\right)$ is the $j$-th vertex represented by a state space pair of matrices $(1 \leq j \leq v), v$ number of vertices. The pair $\left(\mathbf{A}^{i}\left(w^{i}\right), \mathbf{B}^{i}\left(w^{i}\right)\right)$ represents the model subject to uncertainty, described by the polytopic set ConvexHull $\left\{\left(\mathbf{A}^{i j}, \mathbf{B}^{i j}\right), j=1, \cdots, v\right\}$ for each mode $i \in I$. The coefficients $w^{i j}$ are unknown and possibly time varying. $v$ is assumed to be the same for the each partition. If the polytopes $\mathbf{W}^{i}$ have different number of vertices, $v$ will be chosen for uniformity purposes as $v=\max _{i=1 \cdots s} v_{i}$. In the following, the notation $\mathbf{W}$ will thus be adopted to replace the local polytopes $\mathbf{W}^{i}$.

\section{REACHABILITY AND ATTAINABILITY; A POLYHEDRAL APPROACH}

Let consider the region $\mathbf{R}_{k}, k>1$, as a target region in the global state space $\mathbf{X}$. This section examines the robust one-step control region $\mathbf{R}_{k-1}$ as the region in the state space for which there exist at least one feasible mode $i$ and an admissible control signal able to drive the states from $\mathbf{R}_{k-1}$ into $\mathbf{R}_{k}$ in one-step despite all possible parameter variations, i.e.:

$$
\mathbf{R}_{k-1}=\left\{\begin{array}{c}
\mathbf{x}_{k-1} \in \mathbf{X} \mid \exists i \wedge \mathbf{u}_{k-1} \in \mathbf{U} \quad \text { s.t. } \\
\left\lceil\begin{array}{c}
x_{k-1} \\
u_{k-1}
\end{array}\right] \in \chi_{i} \wedge \\
\mathbf{A}^{i}\left(w_{k-1}^{i}\right) \mathbf{x}_{k-1}+\mathbf{B}^{i}\left(w_{k-1}^{i}\right) \mathbf{u}_{k-1} \in \mathbf{R}_{k}, \\
\forall w_{k-1}^{i} \in \mathbf{W}
\end{array}\right\}
$$

In the following, the computation of this region $\mathbf{R}_{k-1}$ is achieved through a polyhedral approach.

Consider the global state space defined by the following constraints: 


$$
\mathbf{X}:=\left\{\mathbf{F}_{s} \mathbf{x} \leq \mathbf{g}_{s}, \mathbf{F}_{s} \in \mathfrak{R}^{p \times n}, \mathbf{g}_{s} \in \mathfrak{R}^{p}\right\}
$$

The control input is supposed to be bounded:

$$
\mathbf{U}:=\left\{\mathbf{m ~ u} \leq \mathbf{n}, \mathbf{m} \in \mathfrak{R}^{p_{u} \times m}, \mathbf{n} \in \mathfrak{R}^{p_{u} \times 1}\right\}
$$

Let the target region $\mathbf{R}_{k}$ be defined by the following constrains:

$$
\mathbf{R}_{k}:=\left\{\mathbf{F} \mathbf{x}_{k} \leq \mathbf{g}\right\}
$$

Considering the system in the mode $i$ where $i \in(1,2, \cdots, s)$ and the $j$-th vertex state matrix for $1 \leq j \leq v$, and using the system evaluation equation (1), (7) can be rewritten as follows:

$$
\begin{aligned}
& \left\{\mathbf{F}\left(\mathbf{A}^{i j} \mathbf{x}_{k-1}+\mathbf{B}^{i j} \mathbf{u}_{k-1}\right) \leq \mathbf{g}\right\} \\
& \quad \Rightarrow\left\{\mathbf{F A}{ }^{i j} \mathbf{x}_{k-1}+\mathbf{F B}{ }^{i j} \mathbf{u}_{k-1} \leq \mathbf{g}\right\}
\end{aligned}
$$

Looking for the state space domain $\mathbf{R}_{k-1}$, under the $i$ mode and the $j$-th vertex of the uncertain polytopic model, for which there is an feasible input control signal $\mathbf{u}_{k-1}$ that can drive the states from $\mathbf{R}_{k-1}$ to $\mathbf{R}_{k}$ in one step, these inputs can be calculated as follows:

$$
\begin{aligned}
k_{l}^{i j}= & \min _{\mathbf{u}_{k-1}} \mathbf{F B}{ }_{l}^{i j} \mathbf{u}_{k-1} \\
& \text { subject to } \quad \mathbf{m} \mathbf{u}_{k-1} \leq \mathbf{n}
\end{aligned}
$$

where $\mathbf{F B}{ }_{l}^{i j}$ are the rows of $\mathbf{F B}{ }^{i j}$. Let $\mathbf{K}^{i j}$ be a vector of $k_{l}^{i j}$ for all rows of $\mathbf{F B}{ }^{i j}$; then it comes from (8) and (9):

$$
\mathbf{R}_{k-1}^{i j}=\left\{\mathbf{x} \mid \mathbf{F A}{ }^{i j} \mathbf{x}_{k-1} \leq \mathbf{g g}\right\}, \text { where } \quad \mathbf{g g}=\mathbf{g}-\mathbf{K}^{i j}
$$

For polytopic uncertain switched piecewise linear hybrid systems, the state space region $\mathbf{R}_{k-1}$ under the $i$-th mode can be determined by [8]:

$$
\mathbf{R}_{k-1}^{i}=\bigcap_{j=1}^{v_{i}} \mathbf{R}_{k-1}^{i j}
$$

The region in the state space under all subsystems (modes) for which there exist a feasible mode (1) and an admissible control signal able to drive the states from it into the region $\mathbf{R}_{k}$ in one-step despite all possible parameter variations is finally given by the following relation:

$$
\mathbf{R}_{k-1}=\bigcup_{i=1}^{s} \mathbf{R}_{k-1}^{i}
$$

The procedure presented above can be repeated in a recursive way to find the domain for any limited $N$ horizon steps. Using a dynamic programming approach, after defining the target region $\mathbf{R}_{k+N}$, the state space domain $\mathbf{R}_{k}$ can be recursively calculated, that includes all the states having a feasible control policy that can in $N$ steps derive the states to $\mathbf{R}_{k+N}$ despite the parameter variations. 
Remark 1: For piecewise linear hybrid systems with many sub-models $s$ and for long horizon steps $N$, this may implies the exploration of a large number of regions (exponential complexity) (Figure 1-a). Considering "no switch" between sub-models over the $N$ steps horizon (Figure 1-b) leads to the lowest complexity mechanism. For many applications, as will be seen in the application section, this suboptimal approach (Considering no switch) provides good performances.

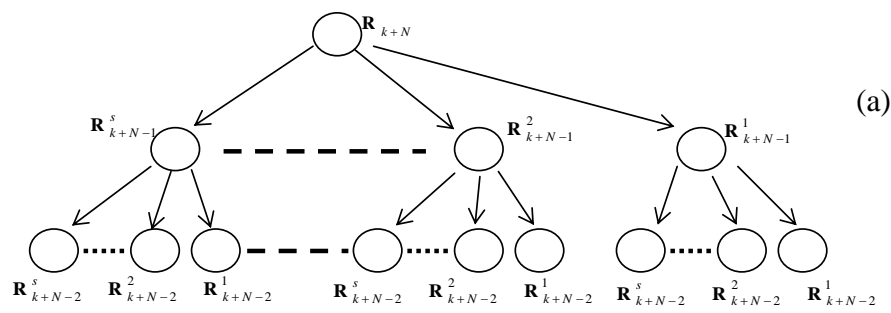

(a)

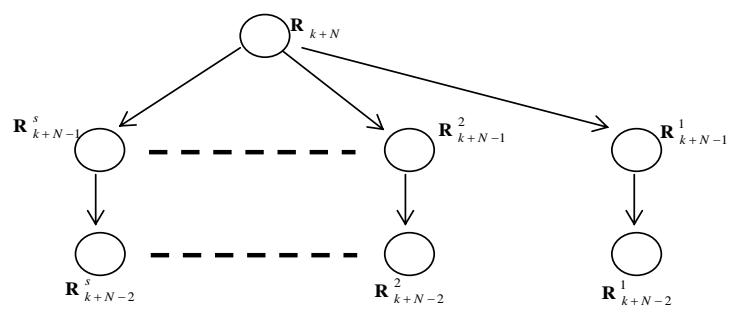

(b)

Fig. 1. Regions exploration, (a) complete exploration, (b) exploration with no switch over the $N$ steps.

Safety, a well-known geometric condition for a set to be safe (control invariant) is the following [11]:

the set $\mathbf{R}_{k+1}$ is safe if and only if $\mathbf{R}_{k+1} \subseteq \mathbf{R}_{k}$

Attainability, given a finite number of regions $\left(\mathbf{R}_{k}, \mathbf{R}_{k+1}, \cdots, \mathbf{R}_{k+N}\right) \in I \times \chi$, the attainability for this sequence of regions is equivalent to the following two different properties:

first the direct reachability from region $\mathbf{R}_{k+j}$ to $\mathbf{R}_{k+j+1}$ for $0 \leq j \leq N-1$, secondly the safety (or control invariance) for region $\mathbf{R}_{k+N}$.

\section{ROBUST MODEL PREDICTIVE CONTROL}

The min-max control technique is proposed in the literature as a robust control for such problems, which minimizes the maximum cost, to try to counteract the worst disturbance. This paper focuses on the model predictive control for switched piecewise linear hybrid systems with quadratic cost function as a fast suboptimal robust solution.

\section{General Consideration}

Model predictive control (MPC) has proved to efficiently control a wide range of applications in industry. It is capable to control a great variety of processes, including systems with long delay times, non-minimum phase systems, unstable systems, multivariable systems, and constrained systems [12]. 
The main idea of predictive control is to use a model of the plant to predict future outputs of the system. Based on this prediction, at each sampling period, a sequence of future control values is elaborated through an on-line optimization process, which maximizes the tracking performance while satisfying constraints. Only the first value of this optimal sequence is applied to the plant, the whole procedure is repeated again at the next sampling period according to the 'receding' horizon strategy [13].

The cost function to be minimized is generally a weighted sum of square predicted errors and square future control values, e.g. in Generalized Predictive Control (GPC) [14].

\section{Robust MPC For Switched Uncertain Piecewise Linear Hybrid Systems}

The control object is for the closed-loop system to exhibit certain desired behavior despite the uncertainties. Specifically, given finite number of regions $\left\{\mathbf{R}_{0}, \mathbf{R}_{1}, \cdots, \mathbf{R}_{N}\right\}$ in the state space, the goal is for the closed-loop system trajectories, starting from the given initial region $\mathbf{R}_{0}$, to go through the sequence of finite number of regions $\mathbf{R}_{1}, \mathbf{R}_{2}, \cdots, \mathbf{R}_{N}$ in the desired order and finally reach the final region $\mathbf{R}_{N}$.

The model predictive control proposed here requires solving at each sampling time the following problem:

$$
\begin{aligned}
& \min _{\mathbf{u}_{k}^{k+N-1}} J\left(\mathbf{u}_{k}^{k+N-1}, \mathbf{x}_{k}\right)=\sum_{j=1}^{N}\left\|\mathbf{x}_{k+j}-\mathbf{x}_{e}\right\|_{\boldsymbol{\Lambda}}^{2}+\sum_{j=0}^{N-1} r_{j j}\left\|\mathbf{u}_{k+j}\right\|_{\Gamma}^{2} \\
& \text { s.t. : }\left\{\begin{array}{l}
\mathbf{Q}^{i}\left[\begin{array}{l}
\mathbf{x}_{k+1}=\mathbf{A}^{i}\left(w_{k+j}^{i}\right) \mathbf{x}_{k}+\mathbf{B}^{i}\left(w_{k}^{i}\right) \mathbf{u}_{k}, \\
{\left[\mathbf{u}_{k+j}\right.}
\end{array}\right] \leq \mathbf{q}^{i} \\
\mathbf{u}_{k+j-1} \in \mathbf{U}, \\
\mathbf{x}_{k+j} \in \mathbf{R}_{k+j}, \quad \text { for } \quad j=1,2, \cdots, N
\end{array}\right.
\end{aligned}
$$

where $x_{e}$ is the states reference, $\boldsymbol{\Lambda}, \boldsymbol{\Gamma}$ are weighting diagonal matrices.

In order to solve this equation, the model applied at each instant has to be determined. As the future sequence of subsystems over the prediction horizon is unknown, all potential sequences of subsystems $I=\left\{I_{k}, I_{k+1}, \cdots, I_{k+N-1}\right\}$ have to be examined, where $I_{k+j}$ is one mode among the $s$ modes at prediction time $j$, for $j=0,1, \cdots, N-1$. As for each model the value of the logical variable is fixed, the MPC problem is solved by a QP for each potential sequence, providing the optimal control while fulfilling the input and state constraints and the regions sequence $\mathbf{R}_{k+j}$, for $j=0,1, \cdots, N$ as well.

Remark 2: At each sampling time, the decision process can drive the system to any particular feasible mode due to receding horizon implementation of the optimal openloop sequence. To sum up, the conservatism is only related to the feasible set coverage and not directly to the chosen performance index. 
Remark 3: If the initial state $\mathbf{x}_{k}$ is included in the union of regions $\mathbf{R}_{k-N}^{i}$ of different modes $(i)$, the MPC technique can select a suboptimal solution among all feasible modes. The feasibility at instant $k$ implies feasibility at any instant $k+1$ to $k+N$. The longest the prediction, the largest the feasible domain will be.

\section{APPLICATION}

Consider the two-tank benchmark of Figure 2. The system consists of two tanks, filled by a pump acting on tank 1 , continuously manipulated from 0 up to a maximum flow $Q_{1}$. One switching valve $V_{12}$ controls the flow between the tanks, this valve is assumed to be either completely opened or closed ( $V_{12}=1$ or 0 respectively). The $V_{N_{2}}$ manual valve controls the nominal outflow of the second tank. It is assumed in further

simulations that the manual valves, $V_{N 1}$ is always closed and $V_{N_{2}}$ is open. The liquid levels to be controlled are denoted $h_{1}$, and $h_{2}$ for each tank respectively.

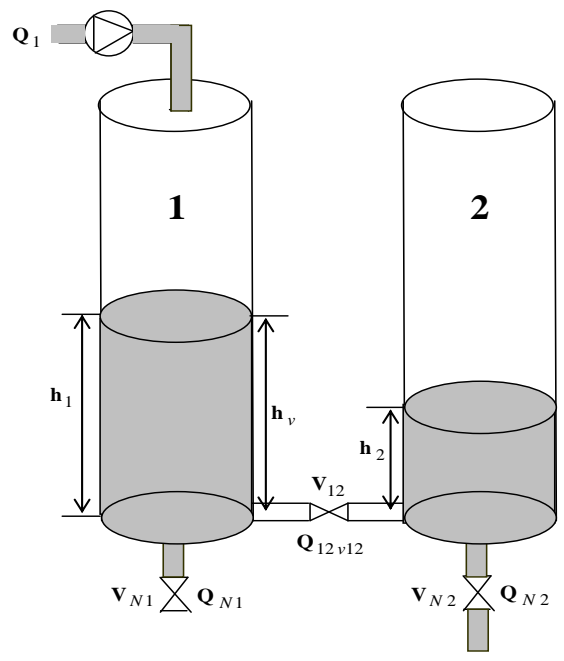

Fig. 2. Two-tank benchmark.

The conservation of mass in the tanks provides the following differential equations:

$$
\begin{aligned}
& \dot{h}_{1}=\frac{1}{A}\left(Q_{1}-Q_{12 V 12}\right) \\
& \dot{h}_{2}=\frac{1}{A}\left(Q_{12 V 12}-Q_{N 2}\right)
\end{aligned}
$$

where the Qs denote the flows and $A$ is the cross-sectional area of each of the tanks. The Toricelli law defines the flows in the valves by following expressions:

$$
\begin{aligned}
& Q_{12 V 12}=V_{12} a S_{12} \operatorname{sign}\left(h_{1}-h_{2}\right) \sqrt{\left|2 g\left(h_{1}-h_{2}\right)\right|} \\
& Q_{N 2}=V_{N 2} a S_{N 2} \sqrt{2 g h_{3}}
\end{aligned}
$$


where $S_{i}$ represent the area of valves $V_{i}$ and $a$ is a constant depending on the liquid. From this, a simplified linear model can be obtained under the form:

$$
\begin{aligned}
& Q_{12 V 12} \approx k_{12} V_{12}\left(h_{1}-h_{2}\right) \\
& Q_{N 2} \approx k_{N 2} V_{N 2} h_{2} \\
& \text { where }: k_{12}=a S_{12} \sqrt{\frac{2 g}{h_{\max }},} \\
& k_{N 2}=a S_{N 2} \sqrt{\frac{2 g}{h_{\max }}}
\end{aligned}
$$

The Euler discretisation technique is used to have the following discrete form :

$$
\begin{aligned}
& h_{1}(k+1)=h_{1}(k)+\frac{T_{s}}{A}\left(Q_{1}(k)-k_{12} V_{12}\left(h_{1}(k)-h_{2}(k)\right)\right. \\
& h_{2}(k+1)=h_{2}(k)+\frac{T_{s}}{A}\left(k_{12} V_{12}\left(h_{1}(k)-h_{2}(k)\right)-k_{N 2} V_{N 2} h_{2}(k)\right)
\end{aligned}
$$

where $T_{s}$ is the sampling time, equal to $10 \mathrm{sec}$.

This benchmark can be considered as a piecewise system of form (1), with two subsystems (two modes) described as follows:

For mode one, the valve $V_{12}$ is open $\left(V_{12}=1\right)$ and two vertices for the uncertainty description are considered:

$$
\begin{aligned}
& \mathbf{A}^{11}=\left[\begin{array}{ll}
0.9188 & 0.0812 \\
0.0812 & 0.8377
\end{array}\right], \quad \mathbf{B}^{11}=\left[\begin{array}{c}
721.5007 \\
0
\end{array}\right] \\
& \mathbf{A}^{12}=\left[\begin{array}{ll}
0.9336 & 0.0664 \\
0.0664 & 0.8672
\end{array}\right], \quad \mathbf{B}^{12}=\left[\begin{array}{c}
590.3188 \\
0
\end{array}\right]
\end{aligned}
$$

For mode two, the valve $V_{12}$ is closed $\left(V_{12}=0\right)$ and two vertices for the uncertainty description are also considered:

$$
\begin{aligned}
& \mathbf{A}^{21}=\left[\begin{array}{cc}
1 & 0 \\
0 & 0.9188
\end{array}\right], \quad \mathbf{B}^{21}=\left[\begin{array}{c}
721.5007 \\
0
\end{array}\right] \\
& \mathbf{A}^{22}=\left[\begin{array}{cc}
1 & 0 \\
0 & 0.9336
\end{array}\right], \quad \mathbf{B}^{22}=\left[\begin{array}{c}
590.3188 \\
0
\end{array}\right]
\end{aligned}
$$

Limitations on the global state space are given through the following relation

$$
\mathbf{X}:=\underbrace{\left[\begin{array}{cc}
1 & 0 \\
0 & 1 \\
-1 & 0 \\
0 & -1
\end{array}\right]}_{\mathbf{F}_{s}} \mathbf{x} \leq \underbrace{\left[\begin{array}{c}
0.62 \\
0.62 \\
0 \\
0
\end{array}\right]}_{\mathbf{g}_{s}}
$$


as well as limitations on the control signal:

$$
\mathbf{U}:=\underbrace{\left[\begin{array}{c}
1 \\
-1
\end{array}\right]}_{\mathbf{m}}\left[Q_{1}\right] \leq \underbrace{\left[\begin{array}{c}
0.0001 \\
0
\end{array}\right]}_{\mathbf{n}}
$$

The target region, to which system states will be derived to, is defined by the following constraints:

$$
\mathbf{R}_{k+N}:=\underbrace{\left[\begin{array}{cc}
1 & 0 \\
0 & 1 \\
-1 & 0 \\
0 & -1
\end{array}\right]}_{\mathbf{F}} \mathbf{x} \leq \underbrace{\left[\begin{array}{c}
0.55 \\
0.25 \\
-0.45 \\
-0.15
\end{array}\right]}_{\mathbf{g}}
$$

The approach presented above is first applied to elaborate the region $\mathbf{R}_{k}$ in the state space which includes the states that can be derived in finite $N$ steps to $\mathbf{R}_{k+N}$ despite the parameter variation. However, a suboptimal approach is used here (see Remark 1) as a compromise with the computational load.

With this assumption, Figure 3 presents the regions for mode one with $N=10$, and Figure 4 for mode two with $N=10$, as well, where the vertical axis corresponds to the sampling time (from 0 to $N$ ). For both modes, the region are presented in Figure 5 with $N=5$. The Multi-Parametric (MPT) toolbox [15] was used to deal with the polyhedral operations; to find the intersection, deleting the redundant constraints and also plotting the polyhedral regions.

The robust model predictive control presented above (13) is applied where the model of state evaluation is chosen to be the epicenter of the state matrix $\frac{1}{2}\left(\mathbf{A}^{i 1}+\mathbf{A}^{i 2}\right)$ for

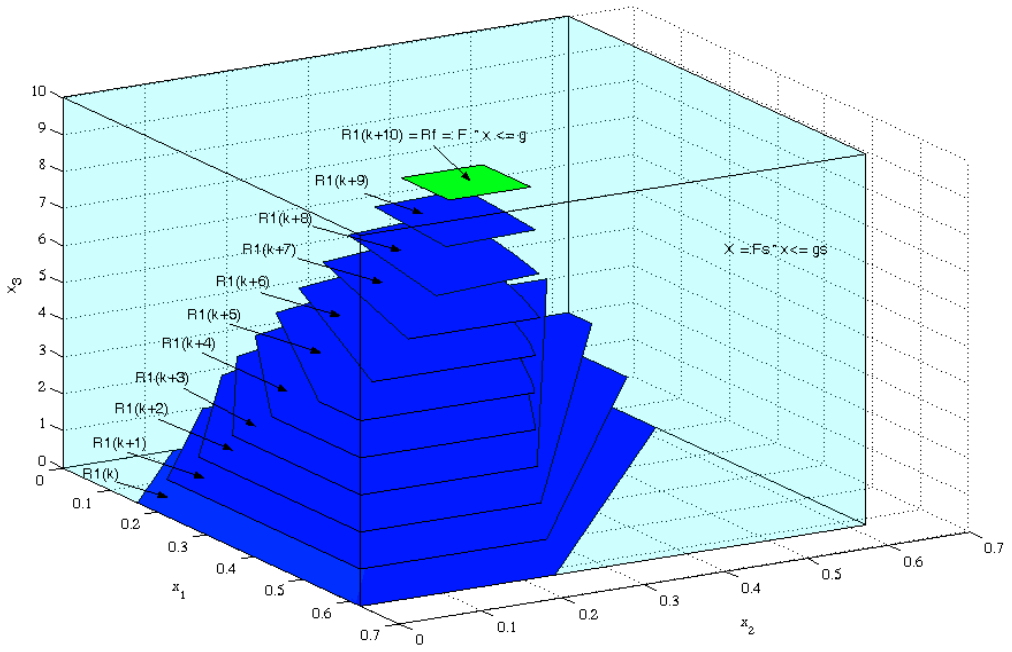

Fig. 3. Regions for mode one with $N=10$ 
each mode. The robust model predictive control is applied so many times, each with different initial states inside the region $\mathbf{R}_{k}$, and in each simulation a random uncertainty $w$, is applied to the system. The weighting diagonal terms in the cost function are chosen such that $\Lambda=1000 \mathbf{I}_{2}$ and $\Gamma=1$, and the states reference is $(0.5,0.2)$.

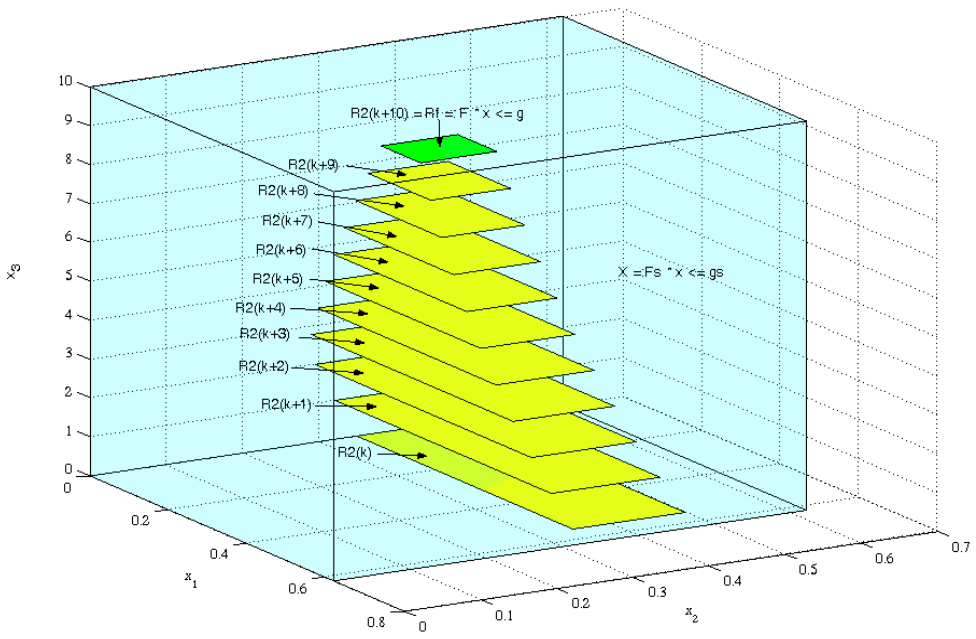

Fig. 4. Regions for mode two with $N=10$.

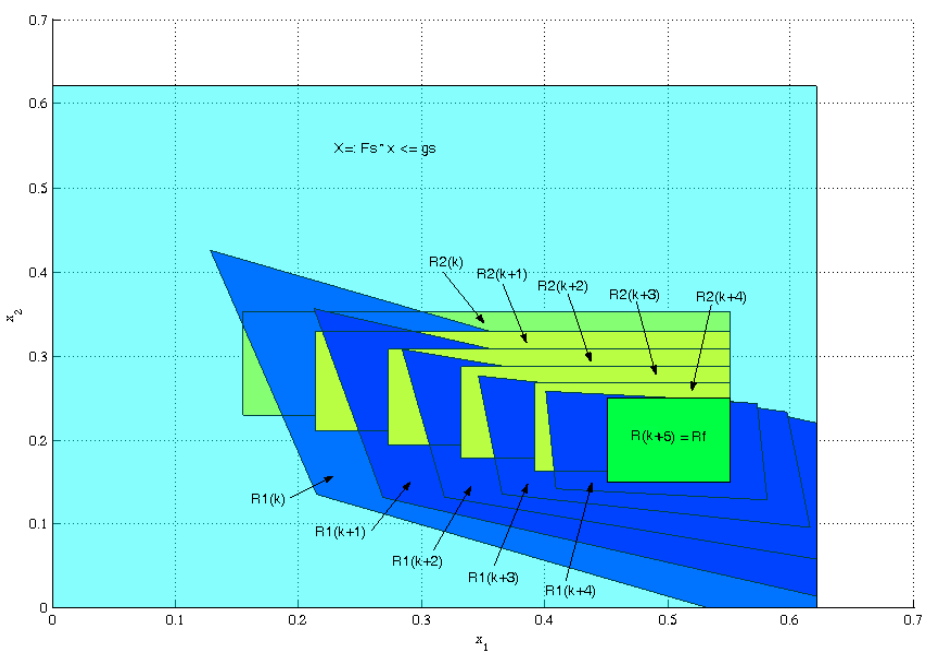

Fig. 5. Regions for both modes with $N=5$

Figure 6 shows some results of robust MPC with $N=3$ for extreme initial states inside $\mathbf{R}_{k}$ with random uncertainty $w$, and as it can be seen on this figure, all the states in $\mathbf{R}_{k}$ are derived in three steps $(N=3)$ to the desired region $\mathbf{R}_{k+3}$ despite the parameter variations. 


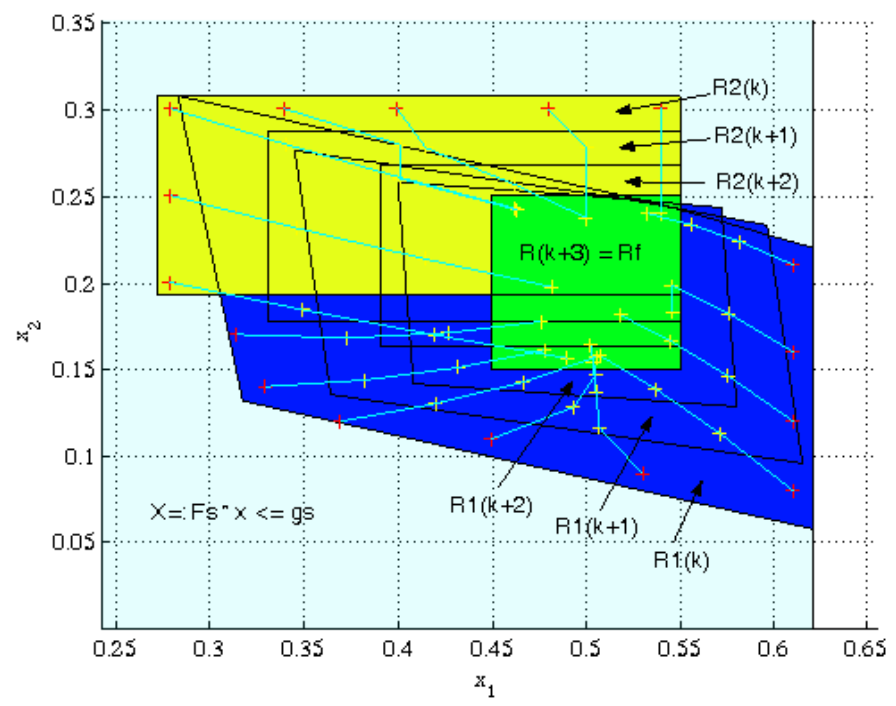

Fig. 6. Robust MPC for different initial states, with $N=3$

\section{CONCLUSION}

This paper has examined a class of uncertain discrete-time piecewise linear hybrid systems with parameter variation, for which a simple polyhedral technique has been proposed to find the regions in the state space where a feasible mode and a robust control is assured to derive the system states to the desired region despite the possible parameter variation. Model predictive control technique has been proposed as a fast and suboptimal robust control for the considered problem.

Future work will consider applying the same techniques on uncertain discrete-time piecewise linear/affine hybrid systems affect by both parameter variation and exterior disturbance.

\section{REFERENCES}

[1] Branicky M.S., V.S. Borkar and S.K. Mitter. (1998) A unified framework for hybrid control: model and optimal control theory. IEEE Transactions on Automatic Control, 43(1):31-45.

[2] Sontag E.D. (1981). Nonlinear regulation: the piecewise linear approach. IEEE Transaction on Automatic Control, 26(2):346-358, April.

[3] Bemporad A. and M. Morari (1999). Control of systems integrating logical, dynamics and constraints. Automatica, 35(3):407-427.

[4] De Schutter B. and T.J.J. van den Boom (2004). Model Predictive Control for Discrete-Event and Hybrid Systems - Part II: Hybrid Systems. Proceedings of the 16th International Symposium on Mathematical Theory of Networks and Systems, B. De Moor and B. Motmans, editor Leuven, Belgium, 5-9 July.

[5] Thomas J., J. Buisson, D. Dumur and H. Guéguen (2003). Predictive Control of Hybrid Systems under a Multi- MLD Formalism. IFAC Conference on Analysis and Design of Hybrid Systems ADHS'03, Saint-Malo, France. 
[6] Olaru S., I. Dumitrache and D. Dumur (2003). Modified MLD form for discrete optimization of hybrid systems, IFAC Conference on Analysis and Design of Hybrid Systems ADHS'03, Saint-Malo, France.

[7] Olaru S., J. Thomas, D. Dumur and J. Buisson (2004). Genetic Algorithm based Model Predictive Control for Hybrid Systems under a Modified MLD Form. International Journal of Hybrid Systems. Vol. 4:1-2.

[8] Hai L. and P.J. Antsaklis (2003). Robust tracking and regulation control of uncertain piecewise linear hybrid systems. ISIS Technical report. http://www.nd.edu/ isis/tech.html.

[9] Necoara I., B. De Schutter, T.J.J. van den Boom and J. Hellendoorn (2004). Predictive control for perturbed continuous piecewise affine systems with pounded disturbances. Proceedings of the 43rd IEEE conference on Decision and Control, Paradise Island, The Bahamas, pp. 1848-1853, December.

[10] Bemporad A., F. Borrelli and M. Morari (2003). Min-Max control of constrained uncertain discrete-time linear systems. IEEE Transactions on automatic control, vol. 48, No. 9,September.

[11] Hai, L. and P.J. Antsaklis (2002). Controller synthesis for a class of uncertain piecewise linear hybrid dynamical systems. Proceedings of the 41st IEEE conference on Decision and Control, Las Vegas, Nevada USA, December.

[12] Camacho E.F. and C. Bordons (1999). Model Predictive Control. Springer.

[13] Dumur, D. and Boucher, P.,1998. A Review Introduction to Linear GPC and Applications. Journal A, 39(4), pp. 21-35.

[14] Clarke, D.W., C. Mohtadi and P.S. Tuffs (1987) Generalized Predictive Control, Part I "The basic algorithm", Part II "Extensions and interpretation". In: Automatica, 23(2): 137-160, March.

[15] Kvasnica M., P. Grieder, M. Baotic and M. Morari (2004). Multi-parametric Toolbox (MPT). ul: http://control.ee.ethz.ch/ mpt/, 2004.

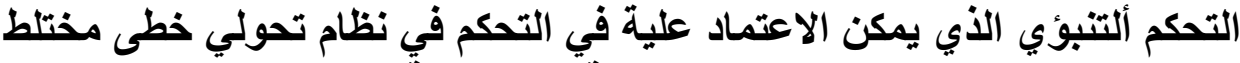 تحكمه معادلات مختلفة طبقا لنقطة العمل فئل}

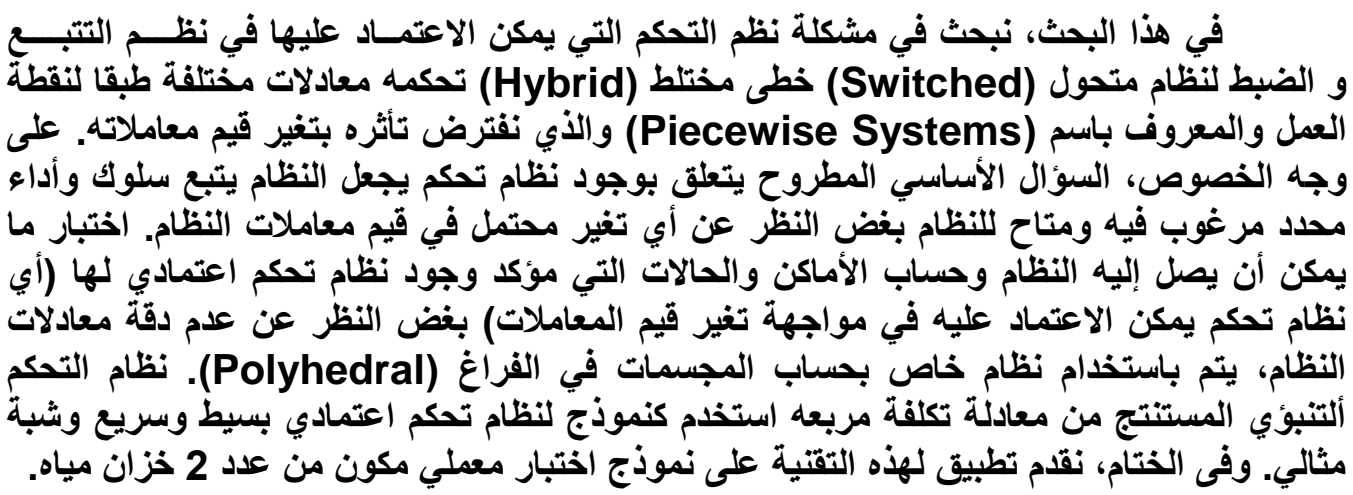

